\title{
Complete genome sequence of Sanguibacter keddieii type strain $\left(\mathrm{ST}-74^{\mathrm{T}}\right)$
}

\author{
Natalia Ivanova ${ }^{1}$, Johannes Sikorski ${ }^{2}$, David Sims ${ }^{1}$, Thomas Brettin ${ }^{1,3}$, John C. Detter ${ }^{1,3}$, Cliff \\ Han $^{1,3}$, Alla Lapidus ${ }^{1}$, Alex Copeland ${ }^{1}$, Tijana Glavina Del Rio ${ }^{1}$, Matt Nolan ${ }^{1}$, Feng Chen ${ }^{1}$, \\ Susan Lucas ${ }^{1}$, Hope Tice ${ }^{1}$, Jan-Fang Cheng ${ }^{1}$, David Bruce ${ }^{1,3}$, Lynne Goodwin ${ }^{1,3}$, Sam Pitluck', \\ Amrita Pati ${ }^{1}$, Konstantinos Mavromatis ${ }^{1}$, Amy Chen ${ }^{4}$, Krishna Palaniappan ${ }^{4}$, Patrik \\ $D^{\prime}$ haeseleer $^{1,5}$, Patrick Chain ${ }^{1,5}$, Jim Bristow ${ }^{1}$, Jonathan A. Eisen ${ }^{1,6}$, Victor Markowitz ${ }^{4}$, Philip \\ Hugenholtz ${ }^{1}$, Markus Göker ${ }^{2}$, Rüdiger Pukall ${ }^{2}$, Hans-Peter Klenk ${ }^{2}$, Nikos C. Kyrpides ${ }^{1 *}$ \\ ${ }^{1}$ DOE Joint Genome Institute, Walnut Creek, California, USA \\ ${ }^{2}$ DSMZ - German Collection of Microorganisms and Cell Cultures GmbH, Braunschweig, \\ Germany \\ ${ }^{3}$ Los Alamos National Laboratory, Bioscience Division, Los Alamos, New Mexico, USA \\ ${ }^{4}$ Biological Data Management and Technology Center, Lawrence Berkeley National Labora- \\ tory, Berkeley, California, USA \\ ${ }^{5}$ Lawrence Livermore National Laboratory, Livermore, California, USA \\ ${ }^{6}$ University of California Davis Genome Center, Davis, California, USA \\ *Corresponding author: Nikos C. Kyrpides
}

Keywords: blood isolate, aerobic, facultative anaerobic, Sanguibacteraceae, Micrococcineae

Sanguibacter keddieii is the type species of the genus Sanguibacter, the only genus within the family of Sanguibacteraceae. Phylogenetically, this family is located in the neighborhood of the genus Oerskovia and the family Cellulomonadaceae within the actinobacterial suborder Micrococcineae. The strain described in this report was isolated from blood of apparently healthy cows. Here we describe the features of this organism, together with the complete genome sequence, and annotation. This is the first complete genome sequence of a member of the family Sanguibacteraceae, and the 4,253,413 bp long single replicon genome with its 3735 protein-coding and 70 RNA genes is part of the Genomic Encyclopedia of Bacteria and Archaea project.

\section{Introduction}

Strain ST-74T (= DSM $10542=$ ATCC $51767=$ JCM $11429=$ NCIMB 703025) is the type strain of Sanguibacter keddieii, which is the type species of the genus Sanguibacter [1]. S. keddieii strain ST-74T was isolated in 1995 by Fernandez-Garayzabal et al. from the blood of apparently healthy dairy cows in Spain [1] as the first member of the genus Sanguibacter and the family of Sanguibacteraceae [2]. On the basis of $16 \mathrm{~S}$ rRNA sequence phylogeny, the small (six species, one genus) family Sanguibacteraceae is located in the neighborhood to the genus Oerskovia [3], now part of the Cellulomonadaceae [2], as well as the Promicromonosporaceae. Here we present a summary classification and a set of features for S. keddieii ST-74 ${ }^{\mathrm{T}}$ together with the description of the complete genomic sequencing and annotation.

\section{Classification and features}

Like strain ST-74T , two more type strains from the genus Sanguibacter $\left(S\right.$. suarezii ST-26 ${ }^{\mathrm{T}}[1]$, and $S$. inulinus [4]) have been isolated from blood of cows. The type strains of the other Sanguibacter species have been isolated from coastal sediment in the Eastern China Sea [5], from surface soil of a ginseng field in South Korea [6], from alpine subnival plants (DQ339590), and from a sea sand sample collected on the Weaver Peninsula on King George Island, Antarctica [7], which may suggest a global ecological versatility of this genus. Only two 
related but yet uncultivated phylotypes with more than 98.5\% 16S rRNA sequence identity were reported from the gastrointestinal tract of pigs (AF371710), and from glacial meltwater at 6,350 $m$ on Mount Everest (EU584523), and no significant matches with any 16S rRNA sequences from environmental genomic samples and surveys are reported at the NCBI BLAST server (March 2009).
Figure 1 shows the phylogenetic neighborhood of $S$. keddieii strain ST-74T in a $16 \mathrm{~S}$ rRNA based tree. Analysis of the four 16S rRNA gene sequences in the genome of strain ST-74T indicated that the genes differ by up to two nucleotides from each other, with two of the copies being identical with the previously published $16 \mathrm{~S}$ rRNA sequence generated from DSM 10542 (X79450).

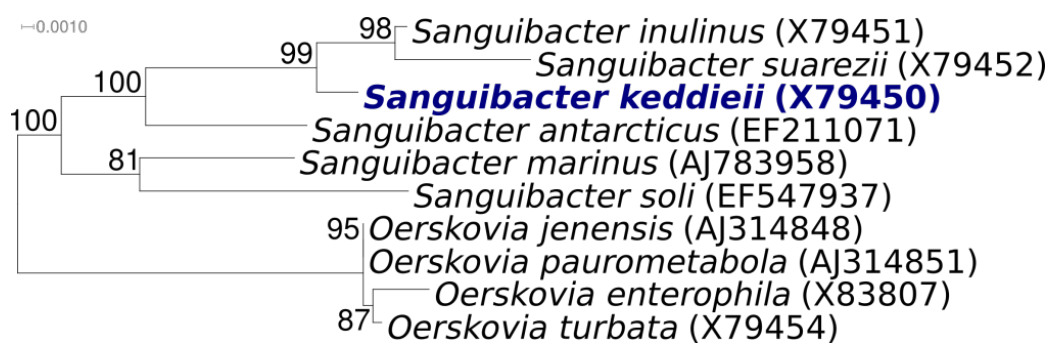

Figure 1. Phylogenetic tree of $S$. keddieii strain ST-74 ${ }^{\top}$ with all type strains of the family Sanguibacteraceae, inferred from 1,468 aligned characters [8] of the $16 \mathrm{~S}$ rRNA sequence under the maximum likelihood criterion $[9,10]$. The tree was rooted with the type strains from the neighbor genus Oerskovia. The branches are scaled in terms of the expected number of substitutions per site. Numbers above branches are support values from 1,000 bootstrap replicates if larger than 60\%. Strains with a genome sequencing project registered in GOLD [11] are printed in blue; published genomes in bold.

S. keddieii ST-74T cells are facultatively anaerobic, Gram-positive, short, irregular shaped motile rods [1] (Table 1 and Figure 2). The colonies on tryptose soy agar (TSA, Difco) are circular, convex, with entire edges and yellow in color. Strain ST-74T is Voges-Proskauer negative and does not reduce nitrate. Casein and gelatin are hydrolyzed. Cellulose and Tween 80 are not hydrolyzed. Acid is produced from a broad range of substrates: $\alpha$ methyl-D-mannoside, $\alpha$-methyl-D-glucoside, $\mathrm{N}$ - acetylglucosamine, amygdalin, rhamnose, $\mathrm{D}$ rafinose, glycerol, L-arabinose, ribose, $\mathrm{D}$-xylose, $\beta$ methyl-xyloside, galactose, glucose, fructose, Dmannose, rhamnose, arbutin, sorbitol, salicin, cellobiose, maltose, lactose, melibiose, sucrose, trehalose, raffinose, glycogen, $\beta$-gentibiose, turanose and lyxose [1]. The optimum growth temperature of strain ST- $74^{\mathrm{T}}$ is $25-30^{\circ} \mathrm{C}$ [1]; it grows at $35^{\circ} \mathrm{C}$ on agar [7] but not at $42^{\circ} \mathrm{C}[1]$.

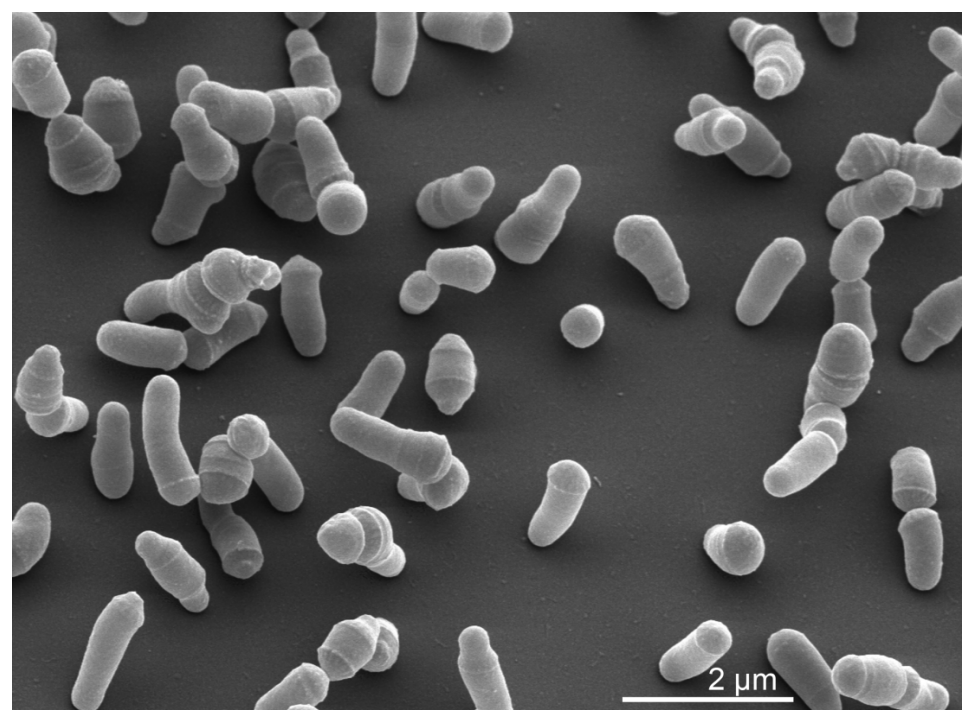

Figure 2. Scanning electron micrograph of S. keddieii ST-74 ${ }^{\mathrm{T}}$ (Manfred Rohde, Helmholtz Centre for Infection Biology, Braunschweig) 


\begin{tabular}{|c|c|c|c|}
\hline MIGS ID & Property & Term & $\begin{array}{l}\text { Evidence } \\
\text { code }\end{array}$ \\
\hline & \multirow{8}{*}{ Current classification } & Domain Bacteria & TAS [13] \\
\hline & & Phylum Actinobacteria & TAS [14] \\
\hline & & Class Actinobacteria & TAS [2] \\
\hline & & Order Actinomycetales & TAS [2] \\
\hline & & Sanguibacteraceae & TAS [15] \\
\hline & & Genus $\quad$ Sanguibacter & TAS [1] \\
\hline & & Species Sanguibacter keddieii & TAS [1] \\
\hline & & Type strain ST-74 & \\
\hline & Gram stain & positive & TAS [1] \\
\hline & Cell shape & short, irregular rods & TAS [1] \\
\hline & Motility & motile & TAS [1] \\
\hline & Sporulation & not reported & \\
\hline & Temperature range & mesophilic & TAS [1] \\
\hline & Optimum temperature & $25-30^{\circ} \mathrm{C}$ & TAS [1] \\
\hline & Salinity & not reported & \\
\hline \multirow[t]{3}{*}{ MIGS-22 } & Oxygen requirement & $\begin{array}{l}\text { primarily aerobe; facultatively anaerobic; no } \\
\text { nitrate reduction }\end{array}$ & TAS [1] \\
\hline & Carbon source & broad variety of sugars & TAS [1] \\
\hline & Energy source & carbohydrates & NAS \\
\hline MIGS-6 & Habitat & animal blood & TAS [1] \\
\hline MIGS-15 & Biotic relationship & free living & NAS \\
\hline \multirow[t]{3}{*}{ MIGS-14 } & Pathogenicity & none & NAS \\
\hline & Biosafety level & 2 & TAS [16] \\
\hline & Isolation & blood of apparently healthy cow & TAS [1] \\
\hline MIGS-4 & Geographic location & Spain & NAS \\
\hline MIGS-5 & Sample collection time & before 1995 & TAS [1] \\
\hline MIGS-4.1 & Latitude, Longitude & not reported & \\
\hline MIGS-4.2 & & & \\
\hline MIGS-4.3 & Depth & not reported & \\
\hline MIGS-4.4 & Altitude & not reported & \\
\hline
\end{tabular}

Evidence codes - IDA: Inferred from Direct Assay (first time in publication); TAS: Traceable Author Statement (i.e., a direct report exists in the literature); NAS: Non-traceable Author Statement (i.e., not directly observed for the living, isolated sample, but based on a generally accepted property for the species, or anecdotal evidence). These evidence codes are from the Gene Ontology project [17]. If the evidence code is IDA, then the property was directly observed for a living isolate by one of the authors or another expert mentioned in the acknowledgements.

Little is known about the chemotaxonomy of strain ST-74T. The major cellular fatty acids are saturated straight chain and branched-chain forms. In strain ST-74T, the straight chain fatty acids 16:0 (53.3\%), 18:0 (10.1\%), 14:0 (5.8\%) predominate over lower amounts of branched chain anteiso-15:0 (11.4\%) and iso-16:0 (5.4\%) fatty acids. This is in contrast to other species in the genus Sanguibacter and in the neighboring Oerskovia and Cellulomonadaceae, where branched chain fatty acids are predominant [18]. Only traces of unsaturated acids, anteiso-15:1 (1.6\%), and no mycolic acids were detected [1], as in the neighboring taxa Oerskovia and other members of Cellulomonadaceae. The murein of $S$. keddieii contains L-Lys-Ser-D-Glu, variation A $4 \alpha$ [1], strikingly different from members of the genus Oerskovia and other members of the family Cellulomonadaceae [1]. Menaquinones are the sole respiratory lipoquinones present, with a partially saturated menaquinone containing nine-isoprene subunits $\mathrm{MK}-9\left(\mathrm{H}_{4}\right)$ predominating [1]. The location of the points of unsaturation are in the second and third isoprene units, adjacent to the napthoquinone nucleus (MK-9 (II, III-H ${ }_{4}$ ), in O. turbata. The phospholipid composition has 
not been reported, but phosphatidylglycerol, diphosphatidylglycerol, phosphatidylinositol, together with phosphoglycolipids have been reported in members of the neighboring taxa Oerskovia and other members of the Cellulomonadaceae [18].

Table 2. Genome sequencing project information

\begin{tabular}{lll}
\hline MIGS ID & Property & Term \\
\hline MIGS-31 & Finishing quality & $\begin{array}{l}\text { Finished } \\
\text { Three genomic libraries: two Sanger } \\
\text { libraries }-8 \text { kb pMCL200 and fosmid } \\
\text { pcc1Fos }-\end{array}$ \\
& Libraries used & $\begin{array}{l}\text { and one 454 pyrosequence standard } \\
\text { library }\end{array}$ \\
& & ABI3730, 454 GS FLX \\
MIGS-29 & Sequencing platforms & $10.4 \times$ Sanger; 20× pyrosequence \\
MIGS-31.2 & Sequencing coverage & Newbler version 1.1.02.15, phrap \\
MIGS-30 & Assemblers & Genemark 4.6b, tRNAScan-SE-1.23, \\
MIGS-32 & Gene calling method & infernal 0.81 \\
& & 19711 \\
& INSDC / Genbank ID & August 30, 2009 \\
& Genbank Date of Release & Gc01087 \\
& GOLD ID & 19711 \\
& NCBI Project ID & 2500901759 \\
& Database: IMG-GEBA & DSM 10542 \\
MIGS-13 & Source material identifier & Tree of Life, GEBA \\
& Project relevance &
\end{tabular}

\section{Genome sequencing and annotation Genome project history}

This organism was selected for sequencing on the basis of its phylogenetic position, and is part of the Genomic Encyclopedia of Bacteria and Archaea project. The genome project is deposited in the Genome OnLine Database [11] and the complete genome sequence in GenBank. Sequencing, finishing and annotation were performed by the DOE Joint Genome Institute (JGI). A summary of the project information is shown in Table 2.

\section{Growth conditions and DNA isolation}

S. keddieii ST-74T, DSM10542, was grown in DSMZ medium 92 (3\% trypticase soy broth, $0.3 \%$ yeast extract) [19] at $30^{\circ} \mathrm{C}$. DNA was isolated from 1-1.5 $\mathrm{g}$ of cell paste using Qiagen Genomic 500 DNA Kit (Qiagen, Hilden, Germany) following the manufacturer's protocol, but with extended (one hour) incubation at $37^{\circ} \mathrm{C}$ as described in Wu et al. [20

\section{Genome sequencing and assembly}

The genome was sequenced using a combination of Sanger and 454 sequencing platforms. All general aspects of library construction and sequencing can be at found the JGI website (http://www.jgi.doe.gov). 454 Pyrosequencing reads were assembled using the Newbler assemb- ler (Version 1.1.02.15, Roche). Large Newbler contigs were broken into 4,746 overlapping fragments of $1,000 \mathrm{bp}$ and entered into assembly as pseudo-reads. The sequences were assigned quality scores based on Newbler consensus q-scores with modifications to account for overlap redundancy and to adjust inflated q-scores. A hybrid 454/Sanger assembly was made using the parallel phrap assembler (High Performance Software, LLC). Possible mis-assemblies were corrected with Dupfinisher [21] or transposon bombing of bridging clones (Epicentre Biotechnologies, Madison, WI). Gaps between contigs were closed by editing in Consed, custom primer walking, or PCR amplification. A total of 2,397 Sanger finishing reads were produced to close gaps, to resolve repetitive regions, and to raise the quality of the finished sequence. The error rate of the completed genome sequence was less than 1 in 100,000. Together all sequence types provided $30.4 \times$ coverage of the genome.

\section{Genome annotation}

Genes were identified using GeneMark [22] as part of the genome annotation pipeline in the Integrated Microbial Genomes Expert Review (IMG-ER) system [23], followed by a round of manual curation using the JGI GenePRIMP pipeline 
(http://geneprimp.jgi-psf.org) [24]. The predicted coding sequences (CDS)s were translated and used to search the National Center for Biotechnology Information (NCBI) nonredundant database, UniProt, TIGRFam, Pfam, PRIAM, KEGG, COG, and InterPro databases. The tRNAScanSE tool [25] was used to find tRNA genes, whereas ribosomal RNAs were found by using the tool RNAmmer [26]. Other non coding RNAs were identified by searching the genome for the Rfam profiles using INFERNAL (v0.81) [27]. Additional gene prediction analysis and manual functional annotation was performed within the Integrated Microbial Genomes (IMG) platform [28].

\section{Metabolic network analysis}

The metabolic Pathway/Genome Database (PGDB) was generated computationally using Pathway Tools software version 12.5 [29] and MetaCyc version 12.5 [30], based on annotated EC numbers and a customized enzyme name mapping file. This metabolic map has undergone no subsequent manual curation and may contain errors, similar to a Tier 3 BioCyc PGDB [31].

\section{Genome properties}

The genome is $4,253,413$ bp long and comprises one main circular chromosome with a $71.9 \%$ GC content (Figure 3 and Table 3). Of the 3,805 genes predicted, 3,735 were protein coding genes, and 70 RNAs. In addition, 25 pseudogenes were identified. The majority of the protein-coding genes (74.4\%) were assigned with a putative function, while those remaining were annotated as hypothetical proteins. The properties and the statistics of the genome are summarized in Table 3. The distribution of genes into COGs functional categories is presented in Table 4. A cellular overview diagram is presented in Figure 4, followed by a summary of metabolic network statistics shown in Table 5.

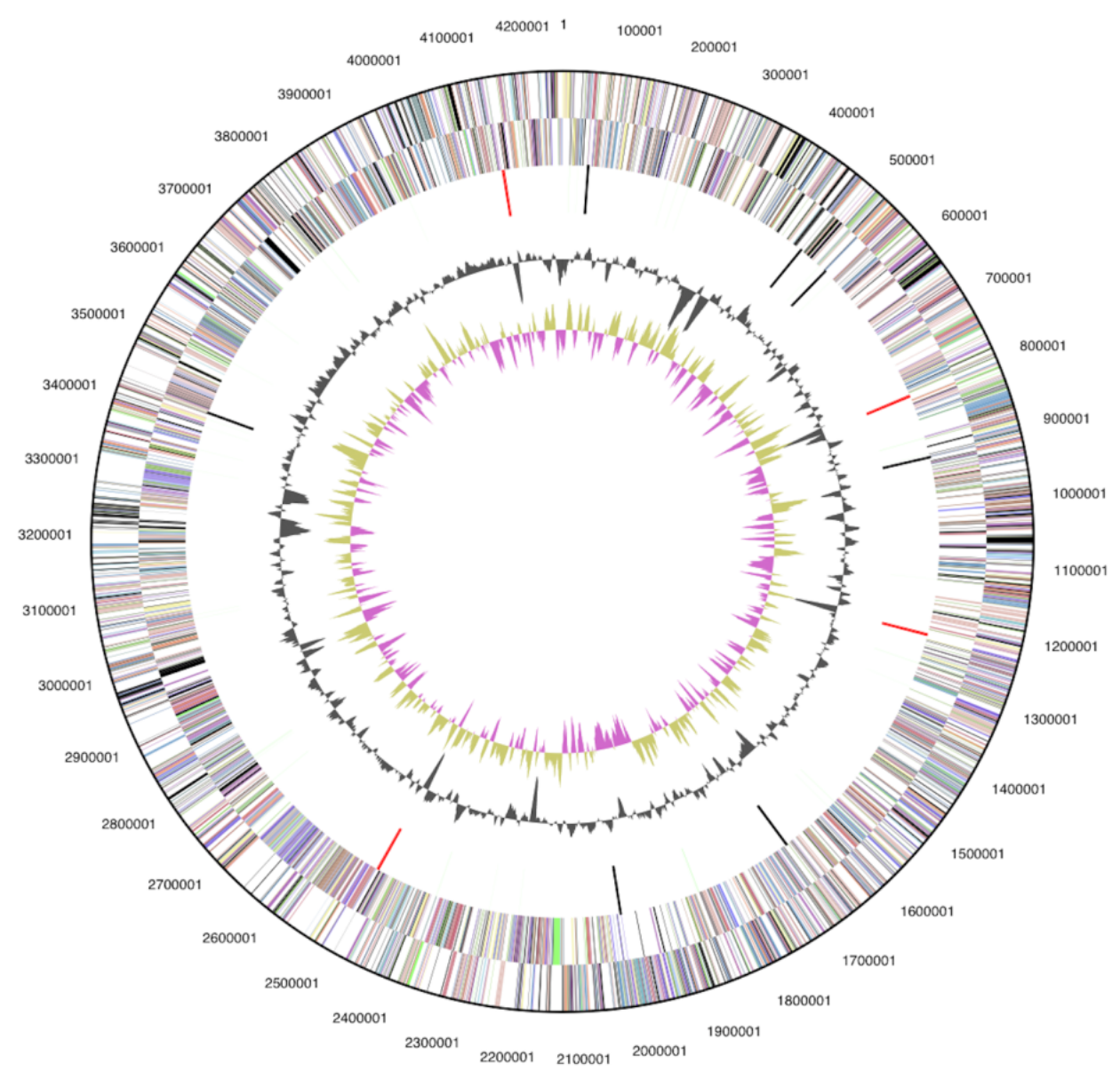

Figure 3. Graphical circular map of the genome. From outside to the center: Genes on forward strand (color by COG categories), Genes on reverse strand (color by COG categories), RNA genes (tRNAs green, rRNAs red, other RNAs black), GC content, GC skew 
Table 3. Genome Statistics

\begin{tabular}{lrr}
\hline Attribute & \multicolumn{1}{c}{ Value } & \% of Total \\
\hline Genome size (bp) & $4,253,413$ & $100.00 \%$ \\
DNA Coding region (bp) & $3,872,139$ & $91.04 \%$ \\
DNA G+C content (bp) & $3,057,630$ & $71.89 \%$ \\
Number of replicons & 1 & \\
Extrachromosomal elements & 0 & \\
Total genes & 3,805 & $100.00 \%$ \\
RNA genes & 70 & $1.84 \%$ \\
rRNA operons & 4 & \\
Protein-coding genes & 3,735 & $98.16 \%$ \\
Pseudo genes & 25 & $0.66 \%$ \\
Genes with function prediction & 2,832 & $74.43 \%$ \\
Genes in paralog clusters & 501 & $13.17 \%$ \\
Genes assigned to COGs & 2,706 & $71.12 \%$ \\
Genes assigned Pfam domains & 2,785 & $73.19 \%$ \\
Genes with signal peptides & 912 & $23.97 \%$ \\
Genes with transmembrane helices & 993 & $26.10 \%$ \\
CRISPR repeats & 0 & \\
\hline
\end{tabular}

Table 4. Number of genes associated with the general COG functional categories

\begin{tabular}{lrrl}
\hline Code & Value & $\begin{array}{r}\text { \% of } \\
\text { total }\end{array}$ & Description \\
\hline J & 166 & 5.0 & Translation \\
A & 1 & 0.0 & RNA processing and modification \\
K & 317 & 10.0 & Transcription \\
L & 120 & 4.0 & Replication, recombination and repair \\
B & 1 & 0.0 & Chromatin structure and dynamics \\
D & 25 & 1.0 & Cell cycle control, mitosis and meiosis \\
Y & 0 & 0.0 & Nuclear structure \\
V & 69 & 2.0 & Defense mechanisms \\
T & 173 & 6.0 & Signal transduction mechanisms \\
M & 134 & 4.0 & Cell wall/membrane biogenesis \\
N & 55 & 2.0 & Cell motility \\
Z & 3 & 0.0 & Cytoskeleton \\
W & 0 & 0.0 & Extracellular structures \\
U & 41 & 1.0 & Intracellular trafficking and secretion \\
O & 84 & 3.0 & Posttranslational modification, protein turnover, chaperones \\
C & 174 & 6.0 & Energy production and conversion \\
G & 354 & 12.0 & Carbohydrate transport and metabolism \\
E & 237 & 8.0 & Amino acid transport and metabolism \\
F & 77 & 3.0 & Nucleotide transport and metabolism \\
H & 119 & 4.0 & Coenzyme transport and metabolism \\
I & 80 & 3.0 & Lipid transport and metabolism \\
P & 199 & 7.0 & Inorganic ion transport and metabolism \\
Q & 43 & 1.0 & Secondary metabolites biosynthesis, transport and catabolism \\
R & 362 & 12.0 & General function prediction only \\
S & 213 & 7.0 & Function unknown \\
- & 1029 & 27.5 & Not in COGs \\
\hline & & & \\
\hline
\end{tabular}




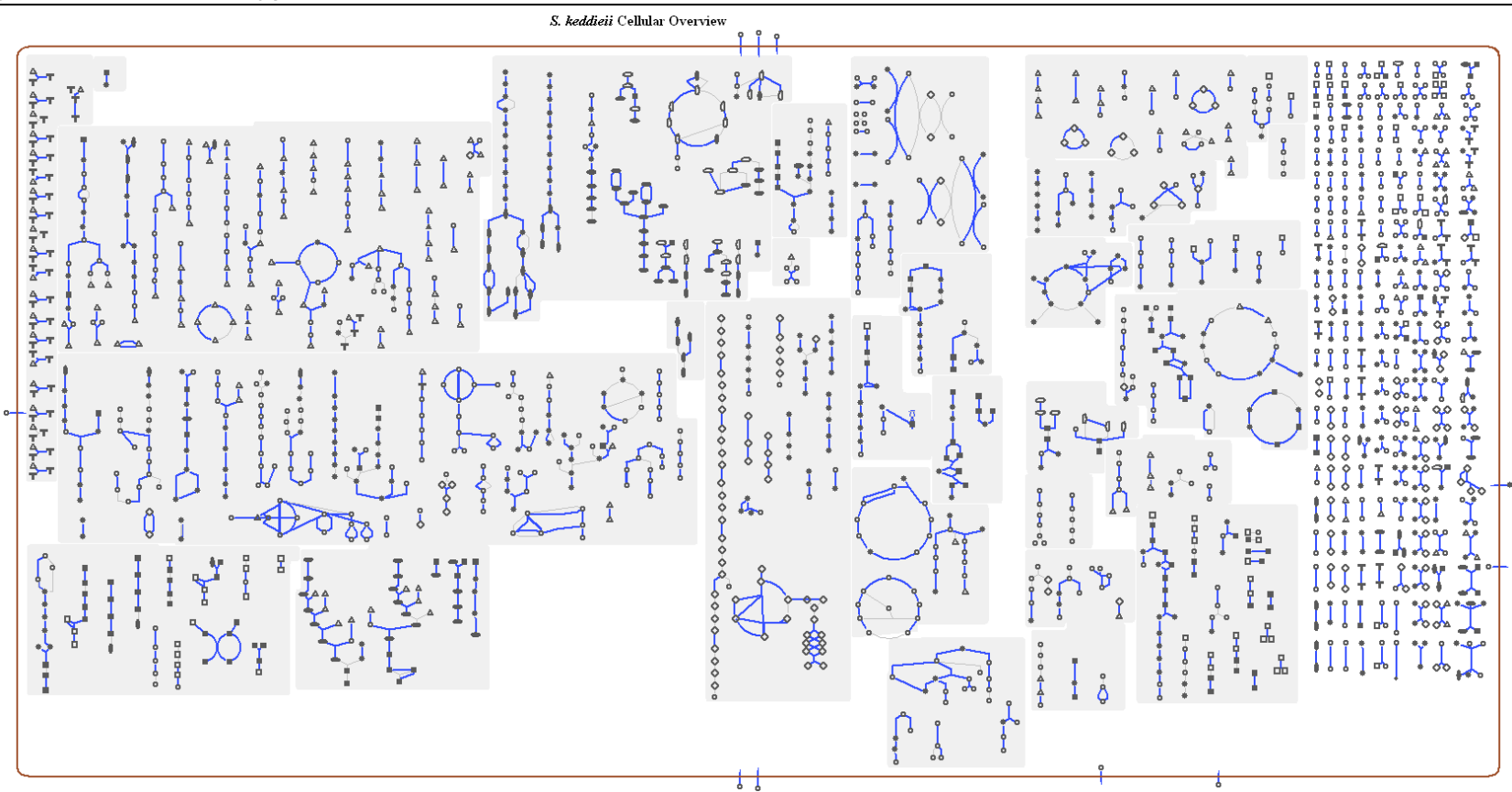

Figure 4. Schematic cellular overview diagram of all pathways of the S. keddieii ST-74 ${ }^{\top}$ metabolism. Nodes represent metabolites, with shape indicating class of metabolite (see key to right). Lines represent reactions.

Table 5. Metabolic Network Statistics

\begin{tabular}{lr}
\hline Attribute & Value \\
\hline Total genes & 3,805 \\
Enzymes & 714 \\
Enzymatic reactions & 935 \\
Metabolic pathways & 205 \\
Metabolites & 676 \\
\hline
\end{tabular}

\section{Acknowledgements}

We would like to gratefully acknowledge the help of Katja Steenblock for growing S. keddieii ST-74 ${ }^{\mathrm{T}}$ cultures, Susanne Schneider for DNA extraction, and Brian J. Tindall for chemotaxonomic advice (all at DSMZ). This work was performed under the auspices of the US Department of Energy Office of Science, Biological and Environmental Research Program, and by the Universi-

\section{References}

1. Fernández-Garayzábal JF, Dominguez L, Pascual C, Jones D, Collins MD. Phenotypic and phylogenetic characterization of some unknown coryneform bacteria isolated from bovine blood and milk: description of Sanguibacter gen.nov. Lett Appl Microbiol 1995; 20:69-75.

PubMed doi:10.1111/j.1472765X.1995.tb01289.x

2. Stackebrandt E, Rainey FA, Ward-Rainey NL. Proposal for a new hierarchic classification system, Actinobacteria classis nov. Int / Syst Bacteriol 1997; 47:479-491. ty of California, Lawrence Berkeley National Laboratory under contract No. DE-AC02-05CH11231, Lawrence Livermore National Laboratory under Contract No. DEAC52-07NA27344, and Los Alamos National Laboratory under contract No. DE-AC02-06NA25396 as well as German Research Foundation (DFG) INST 599/1-1.

3. Prauser H, Lechevalier MP, Lechevalier HA. Description of Oerskovia gen. nov. to harbor Oerskov's motile Nocardia. Appl Microbiol 1970; 19:534. PubMed

4. Pascual C, Collins MD, Grimont PAD, Dominguez L, Fernandes-Garayzabal JF. Sanguibacter inulinus sp. nov. Int J Syst Bacteriol 1996; 46:811813. PubMed

5. Huang Y, Dai X, He L, Wang YN, Wang BJ, Liu Z, Liu SJ. Sanguibacter marinus sp. nov., isolated from coastal sediment. Int I Syst Evol Microbiol 
Ivanova, et al.

2005; 55:1755-1758.

PubMed doi:10.1099/ijs.0.63471-0

6. Kim MK, Pulla RK, Kim SY, Yi TH, Soung NK, Yang DC. Sanguibacter soli sp. nov., isolated from soil of a ginseng field. Int I Syst Evol Microbiol 2008; 58:538-541. PubMed doi:10.1099/ijs.0.65399-0

7. Hong SG, Lee YK, Yim JH, Chun J, Lee HK. Sanguibacter antarcticus sp. nov., isolated from Antarctic sea sand. Int I Syst Evol Microbiol 2008; 58:50-52. PubMed doi:10.1099/ijs.0.65031-0

8. Lee C, Grasso C, Sharlow MF. Multiple sequence alignment using partial order graphs. BioinformatiCs 2002; 18:452-464.

PubMed doi:10.1093/bioinformatics/18.3.452

9. Felsenstein J. Evolutionary trees from DNA sequences: a maximum likelihood approach. J $\mathrm{Mol}$ Evol 1981; 17:368-376. PubMed doi:10.1007/BF01734359

10. Stamatakis A, Hoover P, Rougemont J. A rapid bootstrap algorithm for the RAxML web-servers. Syst Biol 2008; 57:758-771.

PubMed doi:10.1080/10635150802429642

11. Liolios K, Mavromatis K, Tavernarakis N, Kyrpides NC. The Genomes OnLine Database (GOLD) in 2007: status of genomic and metagenomic projects and their associated metadata. Nucleic Acids Res 2008; 36:D475-D479. PubMed doi:10.1093/nar/gkm884

12. Field D, Garrity G, Gray T, Morrison N, Selengut J, Sterk P, Tatusova T, Thomson N, Allen MJ, Angiuoli SV, et al. Towards a richer description of our complete collection of genomes and metagenomes: the "Minimum Information about a Genome Sequence" (MIGS) specification. Nat Biotechnol 2008; 26:541-547.

PubMed doi: $10.1038 /$ nbt1360

13. Garrity GM, Holt J. Taxonomic Outline of the Archaea and Bacteria. Bergey's Manual of Systematic Bacteriology, $2^{\text {nd }} \mathrm{Ed}$. In: G.Garrity GM, Boone DR, Castenholz RW Eds. Vol 1 The Archaea, Deeply Branching and Phototrophic Bacteria. 2001 pp. 155-166

14. Woese CR, Kandler O, Wheelis ML. Towards a natural system of organisms: proposal for the domains Archaea, Bacteria, and Eucarya. Proc Natl Acad Sci USA 1990; 87: 4576-4579.

PubMed doi:10.1073/pnas.87.12.4576

15. Stackebrandt E, Schumann P. Description of Bogoriellaceae fam. nov., Dermacoccaceae fam. nov., Rarobacteraceae fam. nov. and Sanguibac- teraceae fam. nov. and emendation of some families of the suborder Micrococcineae. Int J Syst Evol Microbiol 2000; 50:1279-1285. PubMed

16. Anonymous Biological Agents. Technical rules for biological agents www.baua.de TRBA 466.

17. Ashburner M, Ball CA, Blake JA, Botstein D, Butler H, Cherry JM, Davis AP, Dolinski K, Dwight SS, Eppig JT, et al. Gene ontology: tool for the unification of biology. The Gene Ontology Consortium. Nat Genet 2000; 25:25-29.

PubMed doi:10.1038/75556

18. Minnikin DE, Collins MD, Goodfellow M. Fatty acid and polar lipid composition in the classification of Cellulomonas, Oerskovia ad related taxa. J Appl Bacteriol 1979; 47:87-89.

19. List of growth media used at DSMZ: http://www.dsmz.de/microorganisms/media_list.p $\mathrm{hp}$

20. Wu M, Hugenholtz P, Mavromatis K, Pukall R, Dalin E, Ivanova N, Kunin V, Goodwin L, Wu M, Tindall BJ, et al. A phylogeny-driven genomic encyclopedia of Bacteria and Archaea. Nature 2009; (In press).

21. Han CS, Chain P. Finishing repeat regions automatically with Dupfinisher. In: Proceeding of the 2006 international conference on bioinformatics \& computational biology. Hamid R. Arabnia \& Homayoun Valafar (eds), CSREA Press. June 2629, 2006:141-146.

22. Besemer J, Lomsadze A, Borodovsky M. GeneMarkS: a self-training method for prediction of gene starts in microbial genomes. Implications for finding sequence motifs in regulatory regions. Nucleic Acids Res 2001; 29:2607-2618. PubMed doi:10.1093/nar/29.12.2607

23. Markowitz VM, Mavromatis K, Ivanova NN, Chen IMA, Chu K, Kyrpides NC. Expert IMG ER: A system for microbial genome annotation expert review and curation. Bioinformatics 2009; 25:22712278.

PubMed doi:10.1093/bioinformatics/btp393

24. Pati A, Ivanova N, Mikhailova, N, Ovchinikova G, Hooper SD, Lykidis A, Kyrpides NC GenePRIMP: A Gene Prediction Improvement Pipeline for microbial genomes. (Submitted)

25. Lowe TM, Eddy SR. tRNAscan-SE: a program for improved detection of transfer RNA genes in genomic sequence. Nucleic Acids Res 1997; 25:955-964. PubMed doi:10.1093/nar/25.5.955 
26. Lagesen $\mathrm{K}$, Hallin $\mathrm{P}$, Rødland EA, Staerfeldt $\mathrm{HH}$, Rognes T, Ussery DW. RNAmmer: consistent and rapid annotation of ribosomal RNA genes. Nucleic Acids Res 2007; 35:3100-3108. PubMed doi:10.1093/nar/gkm160

27. Griffiths-Jones S, Moxon S, Marshall M, Khanna A, Eddy SR, Bateman A. Rfam: annotating noncoding RNAs in complete genomes. Nucleic Acids Res 2005; 33:D121-D124. PubMed doi:10.1093/nar/gki081

28. Markowitz VM, Szeto E, Palaniappan K, Grechkin Y, Chu K, Chen IMA, Dubchak I, Anderson I, Lykidis A, Mavromatis K, et al. The Integrated Microbial Genomes (IMG) system in 2007: data content and analysis tool extensions. Nucleic Acids Res 2008; 36:D528-D533.

PubMed doi:10.1093/nar/gkm846
29. Karp PD, Paley SM, Romero P. The Pathway Tools Software. Bioinformatics 2002; 18:S225S232. PubMed

30. Caspi R, Karp P, Foerster H, Fulcher CA, Kaipa P, Krummenacker M, Latendresse M, Paley SM, Rhee SY, Shearer A, et al. The MetaCyc Database of metabolic pathways and enzymes and the BioCyc collection of pathway/Genome Databases. Nucleic Acids Res 2008; 36:D623-D631. PubMed doi:10.1093/nar/gkm900

31. Karp PD, Ouzounis CA, Moore-Kochlacs C, Goldovsky L, Kaipa P, Ahren D, Tsoka S, Darzentas N, Kunin V, Lopez-Bigas N. Expansion of the BioCyc collection of pathway/genome databases to 160 genomes. Nucleic Acids Res 2005; 33:6083-6089. PubMed doi:10.1093/nar/gki892 